\title{
Dynamic Behavior for a Coupled Nonlinear Oscillator Model with Distributed and Discrete Delays
}

\author{
Chunhua Feng
}

\begin{abstract}
In this paper, the oscillatory behavior of the solutions for a coupled nonlinear oscillator model with distributed and discrete delays is investigated. Time delay induced partial death patterns with conjugate coupling in relay oscillators has been investigated in the literature. According to the practical problem, the propagation delays are not only the discrete delays, but also with distributed delay. A model includes distributed and discrete delays is considered. By mathematical analysis method, the oscillatory behavior of the coupled nonlinear oscillator model is brought to the instability of the uniqueness equilibrium point and the boundedness of the solutions. Some sufficient conditions are provided to guarantee the oscillation of the solutions. Computer simulations are given to support the present results. Our simulation suggests that the two theorems are only sufficient conditions.
\end{abstract}

Index Terms - coupled nonlinear oscillator, delay, instability, oscillation.

\section{INTRODUCTION}

It is well known that coupled nonlinear oscillators appear in various settings such as in gene regulatory networks [1], in electronic oscillator circuits [2], in electrical power and traffic flows systems [3], [4], in central pattern generators [5], in mechanical systems $[6,7]$, and many others [8]-[16].

Recently, Roopnarain and Choudhury have investigated the following distributed delay-coupled Stuart-Landau oscillators [17]:

$$
\left\{\begin{array}{c}
x_{1}^{\prime}=x_{1}\left(1-x_{1}^{2}-y_{1}^{2}\right)-\omega y_{1}+k\left(z_{1}-x_{1}\right) \\
y_{1}^{\prime}=y_{1}\left(1-x_{1}^{2}-y_{1}^{2}\right)+\omega x_{1} \\
x_{2}^{\prime}=x_{2}\left(1-x_{2}^{2}-y_{2}^{2}\right)-\omega y_{2}+k\left(z_{2}-x_{2}\right) \\
y_{2}^{\prime}=y_{2}\left(1-x_{2}^{2}-y_{2}^{2}\right)+\omega x_{2} \\
z_{1}^{\prime}=\int_{-\infty}^{t} a x_{2}(\tau) e^{-a(t-\tau)} d \tau-z_{1} \\
z_{2}^{\prime}=x_{1}-z_{2}
\end{array}\right.
$$

where $\omega$ is the intrinsic frequency of each oscillator. The parameter $k$ controls the conjugate coupling strength. By defining $w(t)=\int_{-\infty}^{t} a x_{2}(\tau) e^{-a(t-\tau)} d \tau-z_{1}$, system (1) can be reduced to the following system:

$$
\left\{\begin{array}{c}
x_{1}^{\prime}=x_{1}\left(1-x_{1}^{2}-y_{1}^{2}\right)-\omega y_{1}+k\left(z_{1}-x_{1}\right) \\
y_{1}^{\prime}=y_{1}\left(1-x_{1}^{2}-y_{1}^{2}\right)+\omega x_{1} \\
x_{2}^{\prime}=x_{2}\left(1-x_{2}^{2}-y_{2}^{2}\right)-\omega y_{2}+k\left(z_{2}-x_{2}\right) \\
y_{2}^{\prime}=y_{2}\left(1-x_{2}^{2}-y_{2}^{2}\right)+\omega x_{2} \\
z_{1}^{\prime}=w-z_{1} \\
z_{2}^{\prime}=x_{1}-z_{2} \\
w^{\prime}=a\left(x_{2}-w\right)
\end{array}\right.
$$

The effects of a distributed delay on dynamically coupled
Landau-Stuart limit cycle oscillators are investigated. The linear stability analysis of the system and conditions for Hopf bifurcations that initiate oscillations are also considered Apart from incorporate a distributed delay in coupled Landau-Stuart system, Zhang et al. have discussed discrete time delay in a Stuart-Landau system consisting of three oscillators [18]:

$$
\left\{\begin{array}{c}
x_{j}^{\prime}=\lambda x_{j}-y_{j}+\left(p x_{j}-q y_{j}\right)\left(x_{j}^{2}+y_{j}^{2}\right)+a_{r}\left(x_{j-1, \tau}\right. \\
\left.+x_{j+1, \tau}-2 x_{j, \tau}\right)-a_{l}\left(y_{j-1, \tau}+y_{j+1, \tau}-2 y_{j, \tau}\right) \\
y_{j}^{\prime}=x_{j}+\lambda y_{j}+\left(p x_{j}+q y_{j}\right)\left(x_{j}^{2}+y_{j}^{2}\right)+a_{l}\left(x_{j-1, \tau}\right. \\
\left.+x_{j+1, \tau}-2 x_{j, \tau}\right)+a_{r}\left(y_{j-1, \tau}+y_{j+1, \tau}-2 y_{j, \tau}\right)
\end{array}\right.
$$

where $\quad x_{j, \tau}=x_{j}(t-\tau), y_{j, \tau}=y_{j}(t-\tau), j=1,2,3$. The profile and stability of alternating wave solution system (3), which arises as a bifurcated periodic solution are investigated. The criteria on parameters such that stable alternating wave solutions can be observed are provided. Motivated by the above articles in this paper we shall study the dynamic behavior for the following not only included distributed delay but also involved discrete delay as the following:

$$
\left\{\begin{array}{c}
x_{1}^{\prime}=\lambda_{1} x_{1}-y_{1}+\left(p_{1} x_{1}-q_{1} y_{1}\right)\left(x_{1}^{2}+y_{1}^{2}\right)+a_{11}\left(x_{2 \tau}\right. \\
\left.-x_{1 \tau}\right)+a_{12}\left(x_{3 \tau}-x_{1 \tau}\right)-b_{11}\left(y_{2 \tau}-y_{1 \tau}\right) \\
-b_{12}\left(y_{2 \tau}-y_{1 \tau}\right) \\
y_{1}^{\prime}=x_{1}+\lambda_{1} y_{1}+\left(p_{1} x_{1}+q_{1} y_{1}\right)\left(x_{1}^{2}+y_{1}^{2}\right)+b_{11}\left(x_{2 \tau}\right. \\
\left.-x_{1 \tau}\right)+b_{12}\left(x_{3 \tau}-x_{1 \tau}\right)+a_{11}\left(y_{2 \tau}-y_{1 \tau}\right) \\
+a_{12}\left(y_{2 \tau}-y_{1 \tau}\right) \\
x_{2}^{\prime}=\lambda_{2} x_{2}-y_{2}+\left(p_{2} x_{2}-q_{2} y_{2}\right)\left(x_{2}^{2}+y_{2}^{2}\right)+a_{21}\left(x_{1 \tau}\right. \\
\left.-x_{2 \tau}\right)+a_{22}\left(x_{3 \tau}-x_{2 \tau}\right)-b_{21}\left(y_{1 \tau}-y_{2 \tau}\right) \\
-b_{22}\left(y_{3 \tau}-y_{2 \tau}\right) \\
y_{2}^{\prime}=x_{2}+\lambda_{2} y_{2}+\left(p_{2} x_{2}+q_{2} y_{2}\right)\left(x_{2}^{2}+y_{2}^{2}\right)+b_{21}\left(x_{1 \tau}\right. \\
\left.-x_{2 \tau}\right)+b_{22}\left(x_{3 \tau}-x_{2 \tau}\right)+a_{21}\left(y_{1 \tau}-y_{2 \tau}\right) \\
+a_{22}\left(y_{3 \tau}-y_{2 \tau}\right) \\
x_{3}^{\prime}=\lambda_{3} x_{3}-y_{3}+\left(p_{3} x_{3}-q_{3} y_{3}\right)\left(x_{3}^{2}+y_{3}^{2}\right)+c_{1} x_{4}(t) \\
+a_{32}\left(x_{2 \tau}-x_{3 \tau}\right)-b_{31}\left(y_{1 \tau}-y_{3 \tau}\right)-b_{32}\left(y_{2 \tau}-y_{3 \tau}\right) \\
y_{3}^{\prime}=x_{3}+\lambda_{3} y_{3}+\left(p_{3} x_{3}+q_{3} y_{3}\right)\left(x_{3}^{2}+y_{3}^{2}\right)+b_{31}\left(x_{1 \tau}\right. \\
\left.-x_{3 \tau}\right)+b_{32}\left(x_{2 \tau}-x_{3 \tau}\right)+c_{2} y_{4}(t)+a_{32}\left(y_{2 \tau}-y_{3 \tau}\right) \\
x_{4}^{\prime}(t)=c_{1}\left(x_{1}(t)-x_{4}(t)\right) \\
y_{4}^{\prime}(t)=c_{2}\left(y_{1}(t)-y_{4}(t)\right)
\end{array}\right.
$$

where $\quad x_{4}(t)=\int_{-\infty}^{t} c_{1} x_{1}(\tau) e^{-c_{1}(t-\tau)} d \tau, \quad$ and $\quad y_{4}(t)=$ $\int_{-\infty}^{t} c_{2} y_{1}(\tau) e^{-c_{2}(t-\tau)} d \tau, \quad x_{i \tau}=x_{i}(t-\tau), y_{i \tau}=y_{i}(t-$ $\tau)(i=1,2,3)$. Our goal is to investigate the oscillatory behavior of the solutions for model (4). Noting that the parameters $\lambda_{i}, p_{i}, q_{i}, a_{i j}, b_{i j}$ and $c_{i}$ maybe are different from

Published on July 09, 2021

Chunhua Feng, College of STEM, Alabama State University, USA.

(corresponding e-mail: cfeng @alasu.edu 
each other in system (4). By means of mathematical analysis method, the dynamical behavior of system (4) has been discussed.

\section{PRELIMINARIES}

The system (4) can be expressed in the following matrix form:

$u^{\prime}(t)=P u(t)+Q u(t-\tau)+\phi(u(t))$

where $u(t)=\left[x_{1}, y_{1}, \cdots, x_{4}, y_{4}\right]^{T}, u(t-\tau)=\left[x_{1}(t-\right.$ $\left.\tau), y_{1}(t-\tau), \cdots, x_{4}(t-\tau), y_{4}(t-\tau)\right]^{T}, P$ and $Q$ both are eight by eight matrices, and $\phi(u(t))$ is a eight by one vector:

$P=\left(p_{i j}\right)_{8 \times 8}=\left[\begin{array}{cccccccc}\lambda_{1} & -1 & & 0 & \cdots & 0 & 0 & 0 \\ 1 & \lambda_{1} & & 0 & \cdots & 0 & 0 & 0 \\ 0 & 0 & \lambda_{2} & \cdots & 0 & 0 & 0 \\ \cdots & \cdots & \cdots & \cdots & \cdots & \cdots & \cdots \\ 0 & 0 & 0 & \cdots & \lambda_{3} & 0 & 0 \\ c_{1} & 0 & 0 & 0 & 0 & -c_{1} & 0 \\ 0 & c_{2} & 0 & 0 & 0 & 0 & -c_{2}\end{array}\right]$,
$Q=\left(q_{i j}\right)_{8 \times 8}=\left[\begin{array}{cccccccc}q_{11} & q_{12} & q_{13} & \cdots & q_{16} & 0 & 0 \\ q_{21} & q_{22} & q_{23} & \cdots & q_{26} & 0 & 0 \\ q_{31} & q_{32} & q_{33} & \cdots & q_{36} & 0 & 0 \\ \cdots & \cdots & \cdots & \cdots & \cdots & \cdots & \cdots \\ q_{61} & q_{62} & q_{63} & \cdots & q_{66} & 0 & 0 \\ 0 & 0 & 0 & 0 & 0 & 0 & 0 \\ 0 & 0 & 0 & 0 & 0 & 0 & 0\end{array}\right]$,

where $\quad q_{11}=q_{22}=-\left(a_{11}+a_{12}\right), q_{12}=-q_{21}=b_{11}+$ $b_{12}, q_{13}=a_{11}, q_{14}=-b_{11}, q_{15}=a_{12}, q_{16}=-b_{12}, q_{33}=$ $q_{44}=-\left(a_{21}+a_{22}\right), q_{34}=-q_{43}=b_{21}+b_{22}, \ldots, q_{56}=$ $-q_{65}=b_{31}+b_{32}$,

and

$\phi(u(t))=\left[\left(p_{1} x_{1}-q_{1} y_{1}\right)\left(x_{1}^{2}+y_{1}^{2}\right), \cdots,\left(p_{3} x_{3}+\right.\right.$ $\left.\left.q_{3} y_{3}\right)\left(x_{3}^{2}+y_{3}^{2}\right), 0,0\right]^{T}$.

The linearized system of (5) is

$u^{\prime}(t)=P u(t)+Q u(t-\tau)$

Lemma 1 If matrix $M(=P+Q)$ is a nonsingular matrix for selected parameters, then there exists a unique equilibrium point for system (4) (or (5)).

Proof Assume that $u^{*}=\left[x_{1}^{*}, y_{1}^{*}, \cdots, x_{4}^{*}, y_{4}^{*}\right]^{T}$ is an equilibrium point of system (4) (or (5)), then we have the following algebraic equation

$P u^{*}+Q u^{*}+\phi\left(u^{*}\right)=0$

We first consider

$N u^{*}=0$

where

$$
N=\left(n_{i j}\right)_{8 \times 8}=\left[\begin{array}{cccccccc}
n_{11} & n_{12} & n_{13} & \cdots & n & 0 & 0 \\
n_{21} & n_{22} & n_{23} & \cdots & n_{26} & 0 & 0 \\
n_{31} & n_{32} & n_{33} & \cdots & n_{36} & 0 & 0 \\
\cdots & \cdots & \cdots & \cdots & \cdots & \cdots & \cdots \\
n_{61} & n_{62} & n_{63} & \cdots & n_{66} & 0 & 0 \\
c_{1} & 0 & 0 & 0 & 0 & -c_{1} & 0 \\
0 & c_{2} & 0 & 0 & 0 & 0 & -c_{2}
\end{array}\right]
$$

where $n_{11}=\lambda_{1}-\left(a_{11}+a_{12}\right)+p_{1}\left(x_{1}^{* 2}+y_{1}^{* 2}\right), \quad n_{12}=$ $-1+b_{11}+b_{12}-q_{1}\left(x_{1}^{* 2}+y_{1}^{* 2}\right), n_{13}=a_{11}, n_{14}=-b_{11}$, $n_{15}=a_{12}, n_{16}=-b_{12}, \cdots$,

$n_{21}=1-\left(b_{11}+b_{12}\right)+p_{1}\left(x_{1}^{* 2}+y_{1}^{* 2}\right), \quad n_{22}=\lambda_{1}-$

$\left(a_{11}+a_{12}\right)+q_{1}\left(x_{1}^{* 2}+y_{1}^{* 2}\right), \cdots$,

$n_{66}=\lambda_{3}-a_{32}+q_{3}\left(x_{3}^{* 2}+y_{3}^{* 2}\right)$.

Based on the basic algebraic knowledge, if $N$ is a nonsingular matrix, then system (8) has a unique trivial solution. Namely, $u^{*}=[0,0,0,0,0,0,0,0]^{T}$. However, when $x_{i}^{*}=0, y_{i}^{*}=0(i=1,2,3,4)$, matrix $N$ changes to $M$. The proof is completed.

Lemma 2 If $p_{1}+p_{2}+p_{3}<0, q_{1}+q_{2}+q_{3}<0$, and $\left(p_{1}-q_{1}\right)+\left(p_{2}-q_{2}\right)+\left(p_{3}-q_{3}\right)<0$ for parameters $p_{i}$ and $q_{i}$, then all solutions of system (4) are bounded.

Proof To prove the boundedness of the solutions in system (4), we construct a Lyapunov function

$$
V(t)=\sum_{i=1}^{4} \frac{1}{2}\left(x_{i}^{2}+y_{i}^{2}\right)
$$

Calculating the derivative of $V(t)$ through system (4) we get

$$
\begin{gathered}
\left.V^{\prime}(t)\right|_{(4)}=\sum_{i=1}^{4}\left(x_{i} x_{i}^{\prime}+y_{i} y_{i}^{\prime}\right) \\
\leq \sum_{i=1}^{4}\left(\left|\alpha_{i}\right| x_{i}^{2}+\left|\beta_{i}\right| y_{i}^{2}\right)+\sum_{i \neq j}\left|k_{i j}\right|\left|x_{i} y_{j}\right|+\sum_{i=1}^{3} p_{i} x_{i}^{4} \\
+\sum_{i=1}^{3} q_{i} y_{i}^{4}+\sum_{i=1}^{3}\left(p_{i}+q_{i}\right) x_{i}^{2} y_{i}^{2} \\
+\sum_{i=1}^{3}\left(p_{i}-q_{i}\right)\left(x_{i}^{3} y_{i}+x_{i} y_{i}^{3}\right)
\end{gathered}
$$

where $\alpha_{i}, \beta_{i}$, and $k_{i j}$ are some constants. Obviously, when $x_{i} \rightarrow \infty, y_{i} \rightarrow \infty(1 \leq i \leq 4), x_{i}^{4}, y_{i}^{4}, x_{i}^{3} y_{i}$, and $x_{i} y_{i}^{3} \quad$ are higher order infinity than $x_{i}^{2}, y_{i}^{2}$, and $\left|x_{i} y_{j}\right|$, respectively. Therefore, there exists suitably large $L>0$ such that $\left.V^{\prime}(t)\right|_{(4)}<0$ as $\left|x_{i}\right|>L,\left|y_{i}\right|>L$. This means that all solutions of system (4) are bounded.

\section{OSCILLATION OF THE SOLUTIONS}

Theorem 1 Assume that zero is the unique equilibrium point of system (4) (or (5)) for selecting parameter values. The conditions of Lemma 1 and Lemma 2 are satisfied. Let $\delta_{1}, \delta_{2}, \cdots, \delta_{8}$ and $\sigma_{1}, \sigma_{2}, \cdots, \sigma_{8}$ be characteristic values of matrix $P$ and $Q$, respectively. If the real part of $\delta_{i}$ and $\sigma_{i}$ $(i=1,2, \cdots, 8)$ are negative, then the trivial solution is stable. If there exists some $\operatorname{Re}\left(\delta_{k}\right)>0$, with $\operatorname{Re}\left(\delta_{k}\right)>\left|\operatorname{Re}\left(\sigma_{k}\right)\right|$, or $\operatorname{Re}\left(\delta_{k}\right)<0$, with $\left|\operatorname{Re}\left(\delta_{k}\right)\right|<\operatorname{Re}\left(\sigma_{k}\right)$, then the unique 
equilibrium points of system (4) (or (5)) is unstable. System (4) (or (5)) generates an oscillatory solution.

Proof According to the time delay basic differential equation theory, if all the real part of $\delta_{i}$ and $\sigma_{i}(i=1,2, \cdots, 8)$ are negative, then the trivial solution of system (6) is stable. Noting that $\phi(u(t))$ is a higher order infinitesimal as $x_{i}(t)$ and $y_{i}(t)(i=1,2,3,4)$ tend to zero, therefore, the trivial solution of system (4) (or (5)) is stable. Obviously, if the trivial solution of system (6) is unstable, then the trivial solution of system (4) (or (5)) is also unstable according to the property of delayed differential equation [19]. So, we need to show the instability of the trivial solution of system (6).

Since $\delta_{i}$ and $\sigma_{i}(i=1,2, \cdots, 8)$ are characteristic values of matrix $P$ and $Q$, respectively, then the characteristic equation corresponding to system (6) is the following:

$\prod_{i=1}^{8}\left(\lambda-\delta_{i}-\sigma_{i} e^{-\lambda \tau}\right)=0$

So, we are led to an investigation of the nature of the roots for some $k(k \in\{1,2, \cdots, 8\})$ :

$\lambda-\delta_{k}-\sigma_{k} e^{-\lambda \tau}=0$

System (11) is a transcendental equation which is hard to find all solutions for the equation. However, we show that there exists a positive real part eigenvalue of equation (11) under the assumption of Theorem 1. Let $\lambda=\rho+i \omega, \delta_{k}=$ $\delta_{k 1}+i \delta_{k 2}, \sigma_{k}=\sigma_{k 1}+i \sigma_{k 2}$. If $\operatorname{Re}\left(\delta_{k}\right)>0$, with $\operatorname{Re}\left(\delta_{k}\right)>$ $\left|\operatorname{Re}\left(\sigma_{k}\right)\right|$ holds. Separating the real and imaginary parts from equation (11), we have

$\rho=\delta_{k 1}+\sigma_{k 1} e^{-\rho \tau} \cos (\omega \tau)+\sigma_{k 2} e^{-\rho \tau} \sin (\omega \tau)$

$\omega=\delta_{k 2}-\sigma_{k 1} e^{-\rho \tau} \sin (\omega \tau)+\sigma_{k 2} e^{-\rho \tau} \cos (\omega \tau)$

We show that equation (11) has a positive real part root. Let

$$
f(\rho)=\rho-\delta_{k 1}-\sigma_{k 1} e^{-\rho \tau} \cos (\omega \tau)-\sigma_{k 2} e^{-\rho \tau} \sin (\omega \tau)
$$

Obviously, $f(\rho)$ is a continuous function of $\rho$. Noting that $\operatorname{Re}\left(\delta_{k}\right)>\left|\operatorname{Re}\left(\sigma_{k}\right)\right|$, namely, $\delta_{k 1}>\left|\sigma_{k 1}\right|$. This means that there exists a $\rho_{1}\left(0<\rho_{1} \ll 1\right)$ such that

$$
\begin{gathered}
f\left(\rho_{1}\right)=\rho_{1}-\delta_{k 1}-\sigma_{k 1} e^{-\rho_{1} \tau} \cos (\omega \tau) \\
-\sigma_{k 2} e^{-\rho_{1} \tau} \sin (\omega \tau)<0
\end{gathered}
$$

Noting that $\lim _{\rho \rightarrow \infty} e^{-\rho \tau}=0$, therefore there exists a suitably large $\rho_{2}(>0)$ such that

$$
\begin{aligned}
& f\left(\rho_{2}\right)=\rho_{2}-\delta_{k 1}-\sigma_{k 1} e^{-\rho_{2} \tau} \cos (\omega \tau) \\
& -\sigma_{k 2} e^{-\rho_{2} \tau} \sin (\omega \tau)>0
\end{aligned}
$$

By means of the Intermediate Value Theorem, there exists a $\rho_{0} \in\left(\rho_{1}, \rho_{2}\right)$ such that

$$
\begin{aligned}
& f\left(\rho_{0}\right)=\rho_{0}-\delta_{k 1}-\sigma_{k 1} e^{-\rho_{0} \tau} \cos (\omega \tau) \\
& -\sigma_{k 2} e^{-\rho_{0} \tau} \sin (\omega \tau)=0
\end{aligned}
$$

This means that the characteristic value $\lambda$ has a positive real part $\rho_{0}$. Thus, the trivial solution of system (6) is unstable, implying that the trivial solution of system (4) (or (5)) is unstable. Since all solutions of system (4) are bounded, the instability of the trivial solution and the boundedness of the solutions will force system (4) to generate an oscillatory solution. For the case of $\operatorname{Re}\left(\delta_{k}\right)<0$, with $\left|\operatorname{Re}\left(\delta_{k}\right)\right|<\operatorname{Re}\left(\sigma_{k}\right)$, equation (6) will have a positive root the proof is similar, and we omit it.

Theorem 2 Assume that zero is the unique equilibrium point of system (4) for selecting parameter values. The conditions of Lemma 1 and Lemma 2 are satisfied. If $c_{1}>0$, $c_{2}>0$. Let $n=\max _{1 \leq i \leq 8}\left\{\lambda_{1}+1+c_{1}, \lambda_{1}+1+c_{2}, \lambda_{2}+\right.$ $\left.1, \lambda_{3}+1,-c_{1},-c_{2}\right\} \quad, \quad r=\max _{1 \leq j \leq 8} \sum_{i=1}^{8}\left|q_{i j}\right|$. If the following inequality holds:

$$
n+r>0
$$

Then system (4) has an oscillatory solution.

Proof We still prove that the trivial solution of system (6) is unstable. Let $v(\mathrm{t})=\sum_{i=1}^{4}\left(\left|x_{i}(t)\right|+\left|y_{i}(t)\right|\right)$. From the definition of $n$ and $r$ we have

$$
v^{\prime}(t) \leq n v(t)+r v(t-\tau)
$$

Consider the scalar differential equation

$$
z^{\prime}(t)=n z(t)+r z(t-\tau)
$$

According to the comparison theorem of differential equation, we have $v(\mathrm{t}) \leq z(t)$. For equation (20), the characteristic equation associated with (20) is given by

$\lambda=n+r e^{-\lambda \tau}$

We claim that there exists a positive characteristic root of equation (21). Indeed, let $g(\lambda)=\lambda-n-r e^{-\lambda \tau}$. Then $g(\lambda) i$ s a continuous function of $\lambda$. From condition (18), we have $g(0)=-n-r=-(n+r)<0$. On the other hand, $\lim _{\lambda \rightarrow \infty} e^{-\lambda \tau}=0$. Thus, there exists a suitably large positive $\lambda$ say $\lambda_{0}$ such that $g\left(\lambda_{0}\right)=\lambda_{0}-n-r e^{-\lambda_{0} \tau}>0$. It means that there exists a $\lambda_{*}$ where $\lambda_{*} \in\left(0, \lambda_{0}\right)$ such that $g\left(\lambda_{*}\right)=$ $\lambda_{*}-n-r e^{-\mathrm{e} \lambda_{*} \tau}=0$, again from the Intermediate Value Theorem. In other words, $\lambda_{*}$ is a positive characteristic root of equation (21), implying that the trivial solution of equation (21) is unstable. Since $v(\mathrm{t}) \leq z(t)$, this means that the trivial solution of equation (20), thus the system (6) is unstable. It suggested that system (4) (or (5)) has an oscillatory solution.

\section{Simulation RESUlts}

The simulation is based on the system (4). First the parameters are selected as follows: 
$\lambda_{1}=-3.86, \lambda_{2}=-3.98, \lambda_{3}=-2.95, p_{1}=0.25, p_{2}=$ $-2.85, p_{3}=0.35, q_{1}=-1.96, q_{2}=0.38, q_{3}=$ $0.35, a_{11}=0.45, a_{12}=-0.75, a_{31}=0.55, a_{22}=$ $-0.85, a_{32}=0.35, b_{11}=-3.05, b_{12}=1.65, b_{21}=$ $1.75, b_{22}=-1.95, b_{31}=-2.68, b_{32}=0.50, c_{1}=$ $2.25, c_{2}=2.35$, and the time delay is 0.85 . Then the characteristic values of matrix $P$ are $-2.2500,-2.3500$, $-3.9800 \pm 1.0000 i,-2.9500 \pm 1.0000 i,-4.1500 \pm 1.0000 i$, the characteristic values of matrix $Q$ are 0,0 , $3.4422 \pm 1.7831 i,-3.1934 \pm 2.0089 i, 0.0012 \pm 0.0080 i$. Noting that matrix $Q$ has a characteristic value $3.4422+1.7831 i$ and $3.4422>|-2.9500|$, the conditions of Theorem 1 are satisfied. There exists an oscillatory solution for system (4) (see Fig.1). In order to see the effect of time delay, we change delay as 0.38 , the other parameters are the same as in figure 1, we see that the oscillatory behavior is still maintained. However, the oscillatory amplitude and frequency both are different from each other (see Fig. 2). Then we change the parameters $a_{i j}$ and $b_{i j}$ as $a_{11}=$ 2. $65, a_{12}=-2.35, a_{21}=2.25, a_{22}=-1.25, a_{32}=$ $0.25, b_{11}=-1.65, b_{12}=0.15, b_{21}=-0.25, b_{22}=$ $0.65, b_{31}=0.32, b_{32}=-0.75, c_{1}=1.85, c_{2}=1.75$, time delay is 2.38 , the other parameters are the same as in figure 1. We see that $n=-0.8, r=1.8$ and $n+r>0$. The conditions of Theorem 2 are satisfied. There exists an oscillatory solution of system (4) (see Fig. 3). Then we only change the parameters $c_{1}=1.96, c_{2}=1.98, \lambda_{1}=$ $-3.45, \lambda_{2}=-3.48, \lambda_{3}=-3.65$, the other parameters are the same as in figure 3 , we see that there are oscillatory solutions (see Fig. 4 and Fig. 5). We pointed out that our criterion only is a sufficient condition from the simulation.

(a) Solid line: $x_{1}$, dashed line: $y_{1}$, dotted line: $x_{2}$, dashdotted line: $y_{2}$

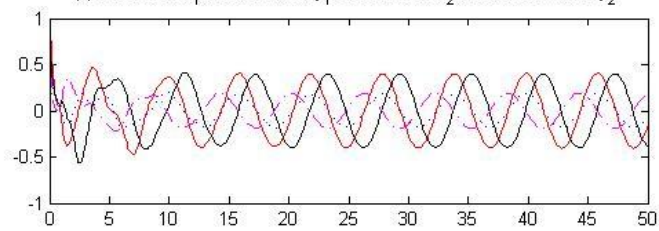

(b) Solid line: $x_{3}$, dashed line: $y_{3}$, dotted line: $x_{4}$, dashdotted line: $y_{4}$

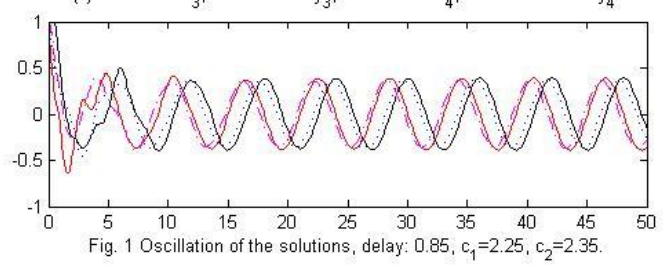

(a) Solid line: $x_{1}$, dashed line: $y_{1}$, dotted line: $x_{2}$, dashdotted line: $y_{2}$,

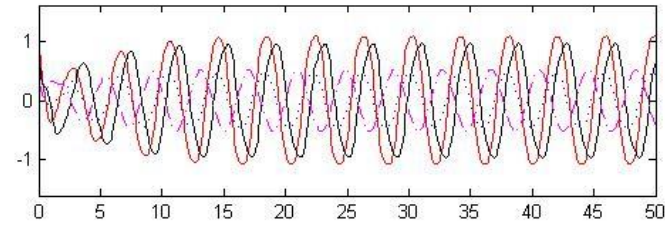

(b) Solid line: $x_{3}$, dashed line: $y_{3}$, dotted line: $x_{4}$, dashdotted line: $y_{4}$

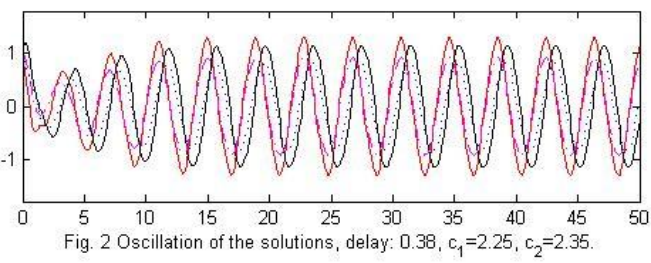

(a) Solid line: $x_{1}$, dashed line: $y_{1}$, dotted line: $x_{2}$, dashdotted line: $y_{2}$.

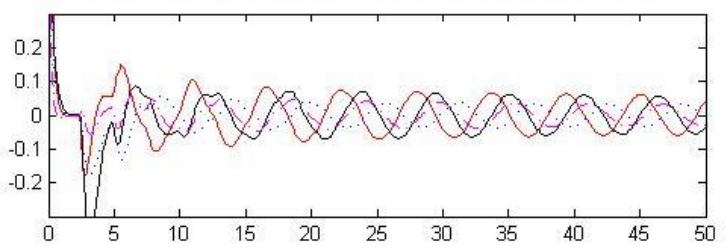

(b) Solid line: $x_{3}$, dashed line: $y_{3}$, dotted line: $x_{4}$, dashdotted line: $y_{4}$

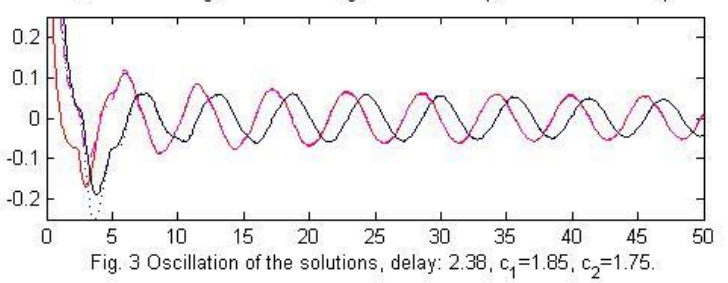

(a) Solid line: $x_{1}$, dashed line: $y_{1}$, dotted line: $x_{2}$, dashdotted line: $y_{2}$.

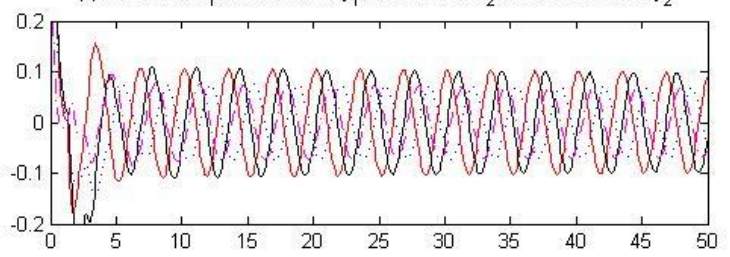

(b) Solid line: $x_{3}$, dashed line: $y_{3}$, dotted line: $x_{4}$, dashdotted line: $y_{4}$

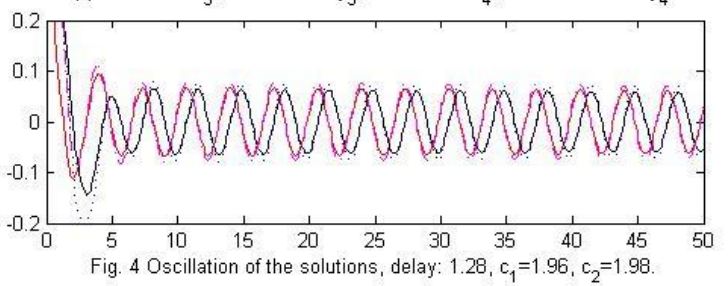

(a) Solid line: $x_{1}$, dashed line: $y_{1}$, dotted line: $x_{2}$, dashdotted line: $y_{2}$

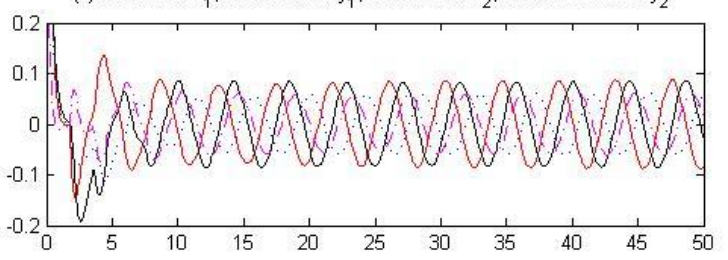

(b) Solid line: $x_{3}$, dashed line: $y_{3}$, dotted line: $x_{4}$, dashdotted line: $y_{4}$

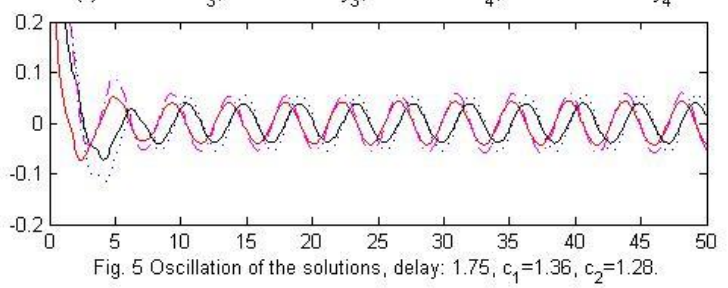

\section{CONCLUSION}

In this paper, we have discussed the oscillatory behavior of the solutions for a coupled nonlinear oscillator model with distributed and discrete delays. Based on mathematical analysis method, we provided some sufficient conditions to guarantee the oscillation of the solutions. Some computer simulations are provided to indicate the results of the criteria. 


\section{REFERENCES}

[1] J. Hasty, D. McMillen, F. Isaacs, and J.J. Collins, "Computational studies of gene regulatory networks," Nature Reviews Genetics, vol. 2, pp. 268-279, 2001

[2] R.D. Ramana, A. Sen, and G.L. Johnston, "Experimental evidence of time-delay-induced death in coupled limit-cycle oscillators," Physical Review Letters, vol. 85, pp. 3381-3384, 2000.

[3] M. Zarifakis, D.J. Byrne, W.T. Coffey, Y.P. Kalmykov, S.V. Titov, and S.J. Carrig, "Comparison of coupled nonlinear oscillator models for the transient response of power generating stations connected to low inertia systems," IEEE Trans. Power Systems, vol. 35, pp. 795-802, 2020.

[4] Z. Zhou, X. Zhang, Q.L. Guo, and H.B. Sun, "Analyzing power and dynamic traffic flows in coupled power and transportation networks," Renewable and Sustainable Energy Reviews, vol. 135, 110083, 2021.

[5] A. Bahramian, A. Nouri, F. Towhidkhah, H. Azarnoush, and S. Jafari, "Introducing a nonlinear coupling for central pattern generator: Improvement on robustness by expanding basin of attraction and performance by decreasing the transient time," J. Vibration and Control, vol. 26, pp. 377-386, 2020.

[6] E.E. Theotokoglou, and D.E. Panayotounakos, "Nonlinear asymptotic analysis of a system of two free coupled oscillators with cubic nonlinearities," Appl. Math. Model. Vol. 43, pp. 509-520, 2017.

[7] J. Taghipour, and M. Dardel, "Steady state dynamics and robustness of a harmonically excited essentially nonlinear oscillator coupled with a two-DOF nonlinear energy sink," Mechanical Systems and Signal Processing, vol. 62, pp. 164-182, 2015.

[8] I. Gowthaman, U. Singh, V.K. Chandrasekar, and D.V. Senthilkumar, "Dynamical robustness in a heterogeneous network of globally coupled nonlinear oscillators," Chaos, Solitons and Fractals, vol. 142, 110396, 2021.

[9] P. Olejnik, and J. Awrejcewicz, "Coupled oscillators in identification of nonlinear damping of a real parametric pendulum," Mech. Syst. Signal Proc. vol. 98, pp. 91-107, 2018.
[10] J. Kyziol, "Metamorphoses of resonance curves for two coupled oscillators: The case of small non-linearities in the main mass frame," Int. J. Non-Linear Mechanics, vol. 76, pp. 164-168, 2015.

[11] R.K. Singh, and T. Bagarti, "Coupled oscillators on evolving networks," Physica D, vol.336, pp. 47-52, 2016.

[12] R. Szalai, and G. Orosz, "Decomposing the dynamics of heterogeneous delayed networks with applications to connected vehicle systems," Phys. Rev. E, vol. 88, 040902, 2013.

[13] M. Tao, "Simply improved averaging for coupled oscillators and weakly nonlinear waves," Commun Nonlinear Sci Numer Simulat, vol. 71 , pp. 1-21, 2019.

[14] E.H. Hellen, and E. Volkov, "How to couple identical ring oscillators to get quasiperiodicity, extended chaos, multistability, and the loss of symmetry," Commun Nonlinear Sci Numer Simulat, vol. 62, pp. 462 479, 2018.

[15] F.K. Hamedani, and G. Karimi, "Design of low phase-noise oscillators based on microstrip triple-band bandpass filter using coupled lines resonator," Microelectronics J. vol. 83, pp. 18-26, 2019.

[16] A. Sharma, "Time delay induced partial death patterns with conjugate coupling in relay oscillators," Physics Letters A, vol. 383, pp. 1865 $1870,2019$.

[17] R. Roopnarain, and S.R. Choudhury, "Amplitude death, oscillation death, and periodic regimes indynamically coupled Landau-Stuart oscillators with and without distributed delay," Math. Comput. Simul. Vol. 187, pp. 30-50, 2021.

[18] S. Zhang, J. Xu, and K.W. Chung, "Stability analysis of alternating wave solution in a Stuart-Landau system with time delay," Commun Nonlinear Sci Numer Simulat, vol. 99, 105808, 2021.

[19] V.B. Kolmanovskii, and A.D. Myshkis, Introduction to the theory and applications of functional-differential equations, Mathematics and its Applications, 463, Kluwer Academic Publishers, Dordrecht, 1999. ch.5. 